Bologna preprint DFUB-92-12

Torino preprint DFTT-92-30

July 1992

\title{
A NEW FAMILY OF DIAGONAL ADE-RELATED SCATTERING THEORIES
}

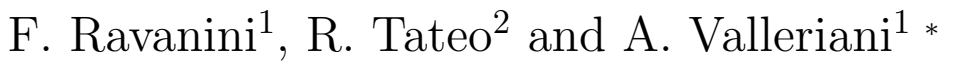 \\ ${ }^{1}$ I.N.F.N. - Sez. di Bologna, and Dip. di Fisica, \\ Università di Bologna, Via Irnerio 46, I-40126 Bologna, Italy \\ ${ }^{2}$ Dip. di Fisica Teorica, Università di Torino \\ Via P.Giuria 1, I-10125 Torino, Italy
}

\begin{abstract}
We propose the factorizable S-matrices of the massive excitations of the nonunitary minimal model $M_{2,11}$ perturbed by the operator $\Phi_{1,4}$. The massive excitations and the whole set of two particle S-matrices of the theory is simply related to the $E_{8}$ unitary minimal scattering theory. The counting argument and the Thermodynamic Bethe Ansatz (TBA) are applied to this scattering theory in order to support this interpretation. Generalizing this result, we describe a new family of non unitary and diagonal $A D E$-related scattering theories. A further generalization suggests the magnonic TBA for a large class of non-unitary $\mathcal{G} \otimes \mathcal{G} / \mathcal{G}$ coset models $\left(\mathcal{G}=A_{\text {odd }}, D_{n}, E_{6,7,8}\right)$ perturbed by $\Phi_{i d, i d, a d j}$, described by non-diagonal S-matrices.
\end{abstract}

*E-mail: ravanini@bologna.infn.it, tateo@torino.infn.it, valleriani@bologna.infn.it 


\section{Introduction}

As pointed out by A.Zamolodchikov [1], some deformations of conformal field theories $(\mathrm{CFT})$ retain an infinite number of integrals of motion even away from criticality where the conformal invariance is broken and a finite correlation length develops. A.Zamolodchikov gave a sufficient condition, now known as the "counting argument" to determine if some combinations of integrals of motion of a given Lorentz spin survive the perturbation of the CFT. If some do survive, and if the perturbed theory is purely massive, then it can be described by a factorizable S-matrix. The values of the spins of the integrals of motion restrict the possible bound state structure and mass ratios in the theory. The bootstrap principle then allows one to actually conjecture a S-matrix of the theory, and give its particle content. To check these conjectures for the S-matrices one can use the fact that the infinite-volume thermodynamics of a massive quantum field theory (QFT) can be expressed in terms of its S-matrix. This method when applied to a factorizable S-matrix theory, leads to the so called Thermodynamic Bethe Ansatz (TBA) [2, 3]

In this letter we use the counting argument and the bootstrap procedure to find the S-matrix of the $M_{2,11}$ minimal model perturbed by its relevant operator $\Phi_{14}$. It turns out that this S-matrix is related to that of the Ising model in a magnetic field and can be encoded on a $E_{8}$ Dynkin diagram. Applying the TBA technique, we confirm that this S-matrix actually describes $M_{2,11}+\Phi_{14}$. The TBA turns out to be encoded on a kind of "product" of graphs described in [12, 10], namely an $E_{8}$ diagram with all nodes filled with tadpoles. At this point, the obvious generalization is to consider the S-matrices related to TBA's encoded on any Dynkin diagram decorated by tadpoles. The general tool to derive these S-matrices is a powerful identity we recently found [10] for the class of $A D E$ S-matrices of [7]. The problem to identify the models corresponding to the newly introduced S-matrices is solved by applying again the TBA method. The result is that these S-matrices describe some non-unitary minimal models of $W$ algebras, perturbed by their field $\Phi_{i d, a d j}$. 
This fact and the form of TBA (or better its encoding on pairs of graphs), suggests a further generalization to entire series of non-unitary coset models of the kind $\mathcal{G} \otimes \mathcal{G} / \mathcal{G}$

\section{Counting argument applied to the $M_{2,11}+\Phi_{1,4}$ minimal non-unitary model}

It is possible to develop a method for finding the lowest values of the conserved spins. This is based on the so called counting argument. Denote by $\hat{\mathbf{V}}_{s+1}$ the space of the quasi-primary descendents of the identity operator at level $s+1$ i.e the factor space

$$
\hat{\mathbf{V}}_{s+1}=\mathbf{V}_{s+1} / \partial_{z} \mathbf{V}_{s}
$$

Analogously, for the family $\mathbf{W}$ of the primary field $\Phi$ we consider the factor space at level $s$.

$$
\hat{\mathbf{W}}_{s+1}=\mathbf{W}_{s+1} / \partial_{z} \mathbf{W}_{s}
$$

The mapping

$$
\partial_{\bar{z}}: \hat{\mathbf{V}}_{s+1} \rightarrow g \hat{\mathbf{W}}_{s}
$$

has a non vanishing kernel if

$$
\operatorname{dim}\left(\hat{\mathbf{V}}_{s+1}\right)>\operatorname{dim}\left(\hat{\mathbf{W}}_{s}\right)
$$

In these circumstances there should exist fields $T_{s+1} \in \hat{\mathbf{V}}_{s+1}$ and $\Phi_{s-1} \in \hat{\mathbf{W}}_{s-1}$ such that:

$$
\partial_{\bar{z}} T_{s+1}=g \partial_{z} \Phi_{s-1}
$$

This imply for the theory with action

$$
A=A^{*}+g \int \Phi(x) d^{2} x
$$




\begin{tabular}{||l|l|l|l|l|l|l|l|l|l||}
\hline 0 & $-\frac{4}{11}$ & $-\frac{7}{11}$ & $-\frac{9}{11}$ & $-\frac{10}{11}$ & $-\frac{10}{11}$ & $-\frac{9}{11}$ & $-\frac{7}{11}$ & $-\frac{4}{11}$ & 0 \\
\hline
\end{tabular}

Table 1: Kac Table of the conformal dimensions $\Delta_{p q}$ of the $M_{2,11}$ model, where $p$ grows in vertical and $q$ in horizontal

(where $A^{*}$ is the UV fixed point action) the existence of a (local) conserved charge with spin s

$$
P_{s}=\int\left(T_{s+1} d z+g \Phi_{s-1} d \bar{z}\right)
$$

The counting argument for the $M_{2,11}$ non-unitary theory perturbed by the operator $\Phi=\Phi_{1,4}$ (see table 1) shows the existence of a conserved current with spin $s=7$ but none with spin $s=3,5$. The fusion rules of this CFT do not have any internal symmetry. These two facts together allow the possibility to have the " $\phi^{3 "}$ and the " $\phi_{1}^{2} \phi_{2}+\phi_{2}^{2} \phi_{1} "$ property [1]. This fixes the mass ratio $\frac{m_{2}}{m_{1}}=\frac{1+\sqrt{5}}{2}$ and the spin spectrum $s=1,7,11,13,17,19,23,29 \bmod (30)$. Now the experience with the bootstrap suggest us to assume that there is another particle with mass ratio $\frac{m_{3}}{m_{1}}=2 \cos \left(\frac{\pi}{30}\right)$ with the property " $\phi_{1}^{2} \phi_{3}$ " and we propose for $S_{11}$ the form

$$
S_{11}=F_{\frac{1}{15}}(\theta) F_{\frac{1}{3}}(\theta) F_{\frac{2}{5}}(\theta) F_{-\frac{1}{30}}(\theta) F_{-\frac{11}{30}}(\theta)
$$

where

$$
\begin{gathered}
f_{x}(\theta)=\frac{\sinh \frac{1}{2}(\theta+i \pi x)}{\sinh \frac{1}{2}(\theta-i \pi x)} \\
F_{x}(\theta)=f_{x}(\theta) f_{i \pi-x}(\theta)=\frac{\tanh \frac{1}{2}(\theta+i \pi x)}{\tanh \frac{1}{2}(\theta-i \pi x)}
\end{gathered}
$$

The remaining scattering amplitudes are obtained by induction, applying the bootstrap equation!

$$
S_{c d}(\theta)=S_{a d}\left(\theta+i \bar{U}_{a c}^{b}\right) S_{b d}\left(\theta-i \bar{U}_{b c}^{a}\right)
$$

\footnotetext{
${ }^{1} \mathrm{~A}$ simple pole of $S_{a b}$ at $\theta_{a b}=i U_{a b}^{c}$ in the direct channel indicates that there exists a bound state $c$ of $a$ and $b$ whose mass is $m_{c}^{2}=m_{a}^{2}+m_{b}^{2}+2 m_{a} m_{b} \cos \left(U_{a b}^{c}\right)$
} 


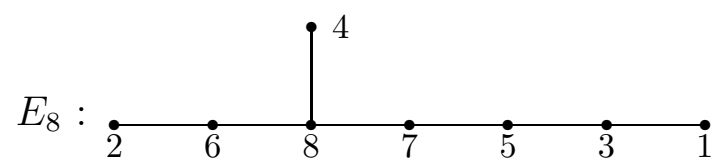

Figure 1: $E_{8}$ Dynkin diagram: the node labelled by $i$ corresponds to the mass $m_{i}$

where $\bar{U}_{a b}^{c}=\pi-U_{a b}^{c}$. Finally the general S-matrix element $S_{a b}$ is given by:

$$
S_{a b}(\theta)=\frac{\tilde{S}_{a b}(\theta)}{\prod_{k} \tilde{S}_{a k}^{G_{b k}^{-1}}(\theta)}
$$

where $\widetilde{S}_{a b}$ is the S-matrix of the Ising model with magnetic field ( the theory $M_{3,4}+$ $\left.\Phi_{1,2}\right)$ and, $G^{-1}$ is the inverse of the incidence matrix of the Dynkin diagram of $E_{8}$.

The exact mass spectrum is (as for $M_{3,4}+\Phi_{1,2}$ ):

$$
\begin{array}{lll}
m_{1}=m & m_{2}=2 m \cos \frac{\pi}{5} & m_{3}=2 m \cos \frac{\pi}{30} \\
m_{4}=2 m_{2} \cos \frac{7 \pi}{30} & m_{5}=2 m_{2} \cos \frac{2 \pi}{15} & m_{6}=2 m_{2} \cos \frac{\pi}{30} \\
m_{7}=m_{2} \cos \frac{\pi}{5} \cos \frac{7 \pi}{30} & m_{8}=4 m_{2} \cos \frac{\pi}{5} \cos \frac{2 \pi}{15} . &
\end{array}
$$

for the correspondence between the particles and the nodes in the Dynkin diagram see fig.1.

\section{TBA for the $M_{2,11}+\Phi_{1,4}$ minimal non-unitary model}

The TBA equations, as they come from the thermodynamic analysis of Bethe wave functions, have the following form:

$$
-\nu_{a}(\theta)+\varepsilon_{a}(\theta)+\frac{1}{2 \pi} \sum_{a}\left[\phi_{a b} * \log \left(1+e^{-\varepsilon_{b}}\right)\right](\theta)=0 \quad, \quad a=1,2, \cdots 8
$$


where $\nu_{a}=R m_{a} \cosh (\theta)$ are the energies (at rapidity $\theta$ ) of particles with the $E_{8}$-related mass spectrum $m_{a}$, and $\varepsilon_{a}(\theta)$ are the so called pseudoenergies, corresponding to each species of particles in the spectrum. The kernel $\phi_{a b}(\theta)$ in eq. (14), encodes the scattering data thanks to its link with the S-matrix and is given by

$$
\phi_{a b}(\theta)=-i \frac{d}{d \theta} \log S_{a b}(\theta)
$$

and the star $*$ stands for the rapidity convolution:

$$
[\phi * L](\theta)=\int_{-\infty}^{+\infty} \phi(\theta-\beta) L(\beta) d \beta
$$

The pseudoenergies $\varepsilon_{a}(\theta)$ determine the Casimir energy $E(R)=\frac{\pi \tilde{c}(R)}{6 R}$ of the field theory on a circle of circumference $R$

$$
\tilde{c}(R)=\frac{3}{\pi^{2}} \sum_{a} \int_{-\infty}^{+\infty} \nu_{a}(\theta) \log \left(1+e^{-\varepsilon_{a}(\theta)}\right) d \theta \quad, \quad \tilde{c}=\tilde{c}(0)=c-24 \Delta_{0}
$$

where $c$ is the central charge and $\Delta_{0}$ the minimal conformal dimension in the Kactable. Now we use the property introduced and proved in [10] for all $A D E \mathrm{~S}$ matrices

$$
\tilde{S}_{a b}\left(\theta+i \frac{\pi}{h}\right) \tilde{S}_{a b}\left(\theta-i \frac{\pi}{h}\right)=e^{-i 2 \pi \Theta(\theta) G_{a b}} \prod_{c} \tilde{S}_{a c}(\theta)^{G_{b c}}
$$

where the term proportional to the step function

$$
\Theta(x)=\lim _{\epsilon \rightarrow 0}\left[\frac{1}{2}+\frac{1}{\pi} \arctan \frac{x}{\epsilon}\right]=\left\{\begin{array}{ccc}
0 & \text { if } & x<0 \\
\frac{1}{2} & \text { if } & x=0 \\
1 & \text { if } & x>0
\end{array}\right.
$$

has to be introduced to take into account the correct prescription for the multivalued function $\log S$ and $h=30$ is the Coxeter number of $E_{8}$. Eq (18), together with (12) shows that $S$ must satisfy the equation

$$
S_{a b}\left(\theta+i \frac{\pi}{h}\right) S_{a b}\left(\theta-i \frac{\pi}{h}\right)=e^{-i 2 \pi \Theta(\theta)\left(G_{a b}-\delta_{a b}\right)} \prod_{c} S_{a c}(\theta)^{G_{b c}}
$$

The property (20), with the relation:

$$
\sum_{b} G_{a b} m_{b}=2 \cos \left(\frac{\pi}{h}\right) m_{a}
$$


implies that the solutions to eqs (14) are also particular solution of the following functional "Y-system"

$$
Y_{a}\left(\theta+\frac{i \pi}{h}\right) Y_{a}\left(\theta-\frac{i \pi}{h}\right)=\prod_{c}\left(1+Y_{c}(\theta)\right)^{G_{a c}}\left(1+\frac{1}{Y_{a}(\theta)}\right)^{-1}
$$

where $Y_{a}(\theta)=e^{\varepsilon_{a}(\theta)}$. Eq (22) has the following periodicity:

$$
Y_{a}(\theta+i \pi P)=Y_{a}(\theta) \quad, \quad P=\frac{h+3}{h}=\frac{11}{10}
$$

This can be shown (along the lines of [4] to be in relation with the conformal dimension of the perturbing field $\Phi$, via the formula

$$
\Delta=1-\frac{2}{P}
$$

This allows to extract in a simple way the parameter $\Delta$, characterizing, together with the effective central charge $\tilde{c}$, the action of the theory. The scaling function energy $F(R)=\frac{R E(R)}{2 \pi}$ expands in a regular series in $R^{\lambda}$ with $\lambda=\frac{4 h}{h+3}$

$$
F(R)=-\frac{\tilde{c}}{12}+\frac{\epsilon_{0} R^{2}}{2 \pi}+\sum_{k=0}^{\infty} f_{k} R^{\lambda k}
$$

where $\epsilon_{0}$ is the coefficient in the bulk energy term and can be calculated from $S_{11}$ [7] and

$$
\tilde{c}=\sum_{a} \frac{6}{\pi^{2}} L\left(\frac{1}{1+y_{a}}\right)
$$

where $L(x)$ is the Rogers dilogarithm function [13]

$$
L=-\frac{1}{2} \int_{0}^{+\infty} d y\left[\frac{\log (y)}{1-y}+\frac{\log (1-y)}{y}\right]
$$

and $y_{a}$ are solutions of the equations

$$
y_{a}^{2}=\prod_{c}\left(1+y_{c}\right)^{G_{a c}}\left(1+\frac{1}{y_{a}}\right)^{-1}
$$

Using the S-matrix elements (12) we find

$$
\tilde{c}=\frac{8}{11}
$$

and from the equation (24)

$$
\Delta=-\frac{9}{11}
$$

i.e the correct values of the effective central charge of the theory $M_{2,11}$ and the conformal dimension of the field $\Phi_{1,4}$ in this theory. 


\subsection{Universal form of TBA}

Now we rewrite eq. (14) in an appealing form which is a generalization of the universal form [4] of the TBA related to the $A D E$ scattering theory. For our purposes we use the identity (20) and take its logarithmic derivative. We find

$$
\phi_{a b}\left(\theta+i \frac{\pi}{h}\right)+\phi_{a b}\left(\theta-i \frac{\pi}{h}\right)=\sum_{c} G_{b c} \phi_{a c}(\theta)-2 \pi \delta(\theta)\left(G_{a b}-\delta_{a b}\right)
$$

Performing a Fourier transform this equation $(k$ is the momentum corresponding to $\theta$ ) becomes

$$
2 \cos \left(\frac{k \pi}{h}\right) \tilde{\phi}_{a b}(k)=\sum_{c} G_{b c} \tilde{\phi}_{a c}(k)-2 \pi\left(G_{a b}-\delta_{a b}\right)
$$

that allows us to rewrite the standard TBA system in the form

$$
\nu_{a}(\theta)=\varepsilon_{a}(\theta)+\frac{1}{2 \pi}\left[\sum_{b} G_{a b} \varphi_{h} *\left(\nu_{b}-\log \left(1+e^{\varepsilon_{b}}\right)\right)+\varphi_{h} * \log \left(1+e^{-\varepsilon_{a}}\right)\right](\theta)
$$

where $\varphi_{h}(\theta)=\frac{h}{2 \cosh \frac{h \theta}{2}}$ is the universal kernel depending only on the Coxeter number $h_{E_{8}}=30$ [4]. We recognize in this form the TBA denoted as $E_{8} \diamond T_{1}$ in [10]

\section{ADET-generalization}

The form of eq. (12) suggests a generalization to all that cases where $G$ is the

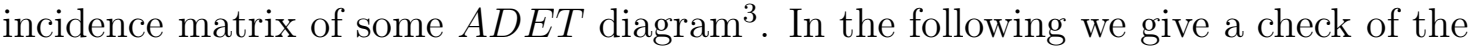
validity of this hypothesis. First of all it is necessary to generalize eq.(12) to the cases where the incidence matrix is not invertible, then we compute the effective central charge for all the models in this class and when $\tilde{c}$ corresponds to a minimal theory we proceed to compare our results with it.

Eq.(12) can be easily transformed using (18) and (20) as

$$
S_{a b}\left(\theta+i \frac{\pi}{h}\right) S_{a b}\left(\theta-i \frac{\pi}{h}\right)=\tilde{S}_{a b}^{-1}(\theta) \tilde{S}_{a b}\left(\theta+i \frac{\pi}{h}\right) \tilde{S}_{a b}\left(\theta-i \frac{\pi}{h}\right) e^{-2 \pi \delta_{a b} \Theta(\theta)}
$$

\footnotetext{
${ }^{2}$ Along the lines of [10] we denote the "tadpole" diagram $A_{2 n} / Z_{2}$ as $T_{n}$, and we refer to that paper for a detailed explanation of the symbol $G \diamond H$.

${ }^{3}$ In the following we will refer to these models as $A D E T \diamond T_{1}$
} 
This equation does not contain explicitly the $G^{-1}$ term, so we can use it both for invertible and non-invertible incidence matrix. Eq.(34) has a simple solution

$$
S_{a b}(\theta)=\tilde{S}_{a b}(\theta) \varphi_{a b}^{-1}(\theta)
$$

where $\varphi$ satisfy the relation

$$
\varphi_{a b}\left(\theta+i \frac{\pi}{h}\right) \varphi_{a b}\left(\theta-i \frac{\pi}{h}\right)=\tilde{S}_{a b}(\theta) e^{2 \pi \delta_{a b} \Theta(\theta)}
$$

The exponential term with the $\Theta$ function means that $\varphi$ has a single pole in $\theta=\frac{i \pi}{h}$. We know that any $A D E T$ diagonal $S$ matrix can always be put in the form [7, 8, 9]

$$
\tilde{S}_{a b}(\theta)=\prod_{\alpha \in \mathcal{A}} f_{\alpha-\frac{1}{h}}(\theta) f_{\alpha+\frac{1}{h}}(\theta)
$$

where $\mathcal{A}$ is a set of rational numbers with common denominator $h$. So $\varphi$ (36) is expressible in terms of such blocks

$$
\varphi_{a b}(\theta)= \pm \prod_{\alpha \in \mathcal{A}} f_{\alpha}(\theta)
$$

The ambiguity in the sign in (38) in the following is fixed by imposing the fermionlike statistic of the system. Of course, when $G$ is invertible $\varphi$ must have the form:

$$
\varphi_{a b}= \pm \prod_{c}\left(\tilde{S}_{a c}\right)^{G_{b c}^{-1}}
$$

Using this prescription the TBA equation and the Y-system can be put in a universal form like (33) and (22) where now $G$ and $h$ are respectively the incidence matrix and the Coxeter number of the corresponding ADET theory.The standard TBA central charge calculation gives

$$
\tilde{c}_{U V}=r\left(1-\frac{h}{h+3}\right)
$$

where $h$ and $r$ are the Coxeter number and the rank of $\mathcal{G}$ respectively. The dimension of the perturbing operator can be easily deduced from the periodicity of the Ysystem:

$$
Y_{a}(\theta+i \pi P)=Y_{\bar{a}}(\theta) \quad, \quad P=\frac{h+3}{h}
$$


In (41) $\bar{a}$ denotes the antiparticle of $a$ and if the diagram does not possess any $Z_{2}$ symmetry $\bar{a}=a$.

We note from 40 that the models denoted as $T_{1} \diamond T_{1}, A_{1} \diamond T_{1}, T_{2} \diamond T_{1}$ and $E_{8} \diamond T_{1}$ have central charge less than 1 , respectively $\tilde{c}=\frac{1}{2}, \frac{3}{5}, \frac{3}{4}, \frac{8}{11}$. Apart from the theory $E_{8} \diamond T_{1}$ studied above, the case $T_{1} \diamond T_{1}$ is the Ising model perturbed with the thermal operator $\Phi_{1,3}$ of dimension $\Delta=\frac{1}{2}$. This is in agreement with the $S_{11}=-1$ matrix obtained using eq (39) and with $\Delta$ obtained from the formula $\Delta=1-\frac{1}{P}$. The next, less trivial model is $A_{1} \diamond T_{1}$. The $S$-matrix for the $A_{1}$ theory is $\tilde{S}_{11}=-1$ and so from (38) and (36) we find for the theory $A_{1} \diamond T_{1}$ :

$$
S_{11}(\theta)=f_{-\frac{1}{2}}(\theta)
$$

and using eqs (24,41) we find $\Delta=\frac{1}{5}$. We identify this model with the minimal non-unitary theory $M_{3,5}+\Phi_{1,3}$ proposed in [5] and studied in [6].

Finally we study the theory $T_{2} \diamond T_{1}$. The theory $T_{2}$ has two particles (it is the theory called $A_{4}^{(2)}$ in [7])

$$
m_{1}=m \quad, \quad m_{2}=m \frac{1+\sqrt{5}}{2}
$$

and S-matrix elements:

$$
\begin{gathered}
\tilde{S}_{11}(\theta)=F_{\frac{2}{5}}(\theta) \\
\tilde{S}_{12}(\theta)=F_{\frac{1}{5}}(\theta) F_{\frac{3}{5}}(\theta) \\
\tilde{S}_{22}(\theta)=F_{\frac{4}{5}}(\theta) F_{\frac{2}{5}}(\theta)^{2}
\end{gathered}
$$

so using (12) it is simple to obtain

$$
\begin{gathered}
S_{11}(\theta)=F_{\frac{2}{5}}(\theta) F_{-\frac{1}{5}}(\theta) \\
S_{12}(\theta)=F_{\frac{1}{5}}(\theta) \\
S_{22}(\theta)=F_{\frac{2}{5}}(\theta)
\end{gathered}
$$

and, thanks to (24), $\Delta=-\frac{1}{4}$. We identify this model as $M_{3,8}+\Phi_{1,3}$. The set of two particle S-matrices is exactly that proposed in [5, 6] 
The remaining $\mathcal{G} \diamond T_{1}$ theories, for $\mathcal{G}=A D E$ also have diagonal S-matrix. For $h$ even, it is not difficult to figure out which are the perturbed CFT's corresponding to these S-matrices. They correspond to some non-unitary minimal models for the $W \mathcal{G}$ algebra, namely $M_{h+1, h+3}^{W \mathcal{G}}$. I The dimension of the perturbing operator, as given by eq.(24), identifies it with the $\Phi_{i d, a d j}$ field. The problem with $h$ odd is that $h+1$ and $h+3$ are not coprime.

This suggests an even more general identification of magnonic TBA's of the form denoted in [10] by $\left(\mathcal{G} \diamond T_{k}\right)_{l}$, with the non-unitary coset models of effective central charge

$$
\tilde{c}_{U V}=\frac{d l}{l+h}\left(1-\frac{l(l+h)}{p(p+2 l)}\right)
$$

where $d=\operatorname{dim} \mathcal{G}$ and $p=h+1, h+2, \ldots$. The UV limit is then described by the models $\mathcal{G}_{k} \otimes \mathcal{G}_{l} / \mathcal{G}_{k+l}$ with $k=\frac{p}{2}-h$. For $l=1$ the series of UV models is the $W \mathcal{G}$ minimal $M_{p, p+2}^{W \mathcal{G}}$. The perturbing operator turns out to be always $\Phi_{i d, i d, a d j}$ as usual. Although these models have non-diagonal S-matrices, their TBA, here conjectured, can give information on the structure of the RG flow of the model off-criticality.

To conclude, we have found a class of diagonal S-matrices related to $A D E$ Lie algebras, definitely different from those studied in [7], that describe the perturbation of certain minimal models of $W$-algebras. Further, we conjecture about a possible extension of the TBA related to this models, to include magnonic structures and allow entire classes of non unitary theories to be followed along their RG flows. We would like to emphasize that all these results have been possible thanks to the high level of sophistication the TBA technique has recently reached, and this is undoubtedly due to its deep and intriguing link with the theory of simply-laced Lie algebras and their Dynkin diagrams first stressed in [4] and pursued in [11, 12, 10]. This observation not only allows an elegant systematization of known results, but also provides a powerful tool for the study of the still mysterious structure of the RG space of actions in two dimensions.

\footnotetext{
${ }^{4}$ We denote here the non-unitary minimal models of $W \mathcal{G}$ by $M_{p q}^{W \mathcal{G}}$, where, as usual, $p$ and $q$ are two coprimes.
} 
Acknowledgements - We are greatly indebted to F.Gliozzi for a lot of very useful discussions. In particular R.T. would like to thank F.Gliozzi for the patient and competent tutoring during this first part of $\mathrm{PhD}$ in Torino. We also are grateful to A.Koubek, G.Mussardo and I.Pesando for useful discussions and help. R.T. thanks the Theory Group at Bologna University for the kind hospitality during the final part of this work.

\section{References}

[1] A.B.Zamolodchikov, Integrable Field Theory from Conformal Field Theory, Proceedings of the Taniguchi Symposium, Kyoto (1988);

A.B.Zamolodchikov, Int. J. Mod. Phys. A4 (1989) 4235

[2] C.N.Yang and C.P.Yang, J. Math. Phys. 10 (1969) 1115

[3] Al.B.Zamolodchikov, Nucl. Phys. B342 (1990) 695

[4] Al.B.Zamolodchikov, Phys. Lett. B253 (1991) 391

[5] F.A.Smirnov, Nucl. Phys. B337 (1990) 156

[6] P.Christe and M.J.Martins, Mod. Phys. Lett. A5 (1990) 26 2189-2195

[7] T.Klassen and E.Melzer, Nucl. Phys. B338 (1990) 485; Nucl. Phys. B350 (1991) 635

[8] P.E.Dorey, Nucl. Phys. B358 (1991) 654

[9] P.Christe and G.Mussardo, Int. J. Mod. Phys. A5 (1990) 469

[10] F.Ravanini, R.Tateo and A.Valleriani, Dynkin TBA's, preprint Bologna DFUB-92-12 and Torino DFTT-31/92 
[11] Al.B.Zamolodchikov, Nucl. Phys. B358 (1991) 497; Nucl. Phys. B358 (1991) 524; Nucl. Phys. B366 (1991) 122;

V.A.Fateev and Al.B.Zamolodchikov, Phys. Lett. B271 (1991) 91

[12] F.Ravanini, Phys. Lett. B282 (1992) 73

[13] L.Lewin, Dilogarithms and associated functions (MacDonald, 1958) 